\title{
Multi-Color Photoluminescence of Terpyridine Crystals
}

\author{
Masaaki Yokota, Atsushi Ito, Norihito Doki \\ Department of Chemistry and Bioengineering, Iwate University, Morioka, Japan \\ Email: myokota@iwate-u.ac.jp
}

Received 25 December 2015; accepted 5 April 2016; published 8 April 2016

Copyright (C) 2016 by authors and Scientific Research Publishing Inc.

This work is licensed under the Creative Commons Attribution International License (CC BY). http://creativecommons.org/licenses/by/4.0/

(c) (i) Open Access

\begin{abstract}
New type of photoluminescence properties of $2,2^{\prime}: 6^{\prime}, 2^{\prime \prime}$-Terpyridine (Terpy) is described. Orthorhombic form of Terpy crystals, which is known to be inherently nonluminous in the visible region, was found to emit strong green light in the presence of traces amount of impurities. Hydroxy-terpyridine, originally included in the Terpy reagent, was found to be the main cause of the green luminescence. Tuning of the luminescent color is possible depending on the dopant species.
\end{abstract}

\section{Keywords}

Terpyridine, Crystal, Photoluminescence, Polymorphism

\section{Introduction}

Photoluminescence of organic crystals is one of the major interests for chemists and crystallographer. Merit of the organic luminescent compounds is that tuning of the color change may be achieved systematically by introducing its analogue and/or by changing molecular packing motif (polymorphism).

Among the various candidates for the organic luminescent compounds proposed until now, we have interested in 2,2':6',2"-Terpyridine (Terpy, Figue 1) as a promising compound for organic luminescence. Until now, two crystal structures of Terpy have been reported as shown in Figure 2 [1] [2]. In orthorhombic form (upper), Terpy molecules are arranged in layers through $\pi-\pi$ interaction, while zigzag arrangement of the molecules are formed in monoclinic form by combination of $\pi-\pi$ and $\mathrm{CH}-\pi$ interactions. The orthorhombic form of Terpy is non-luminescent, while the monoclinic form is luminescent [3]. Transformation of orthorhombic to monoclinic by heating is also reported [3]. The most characteristic point for Terpy is ON-OFF luminescent switching is possible by the solid-state polymorphic transformation [3].

Although, Terpy shows interesting luminescent properties as shown above, the intensity of the emitting light is weak even in case of luminescent monoclinic form. In this study, improvement of the luminescence properties 


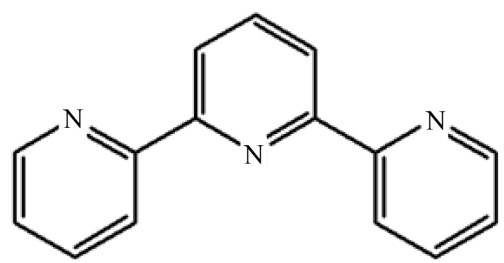

Figure 1. Chemical structure of terpy.
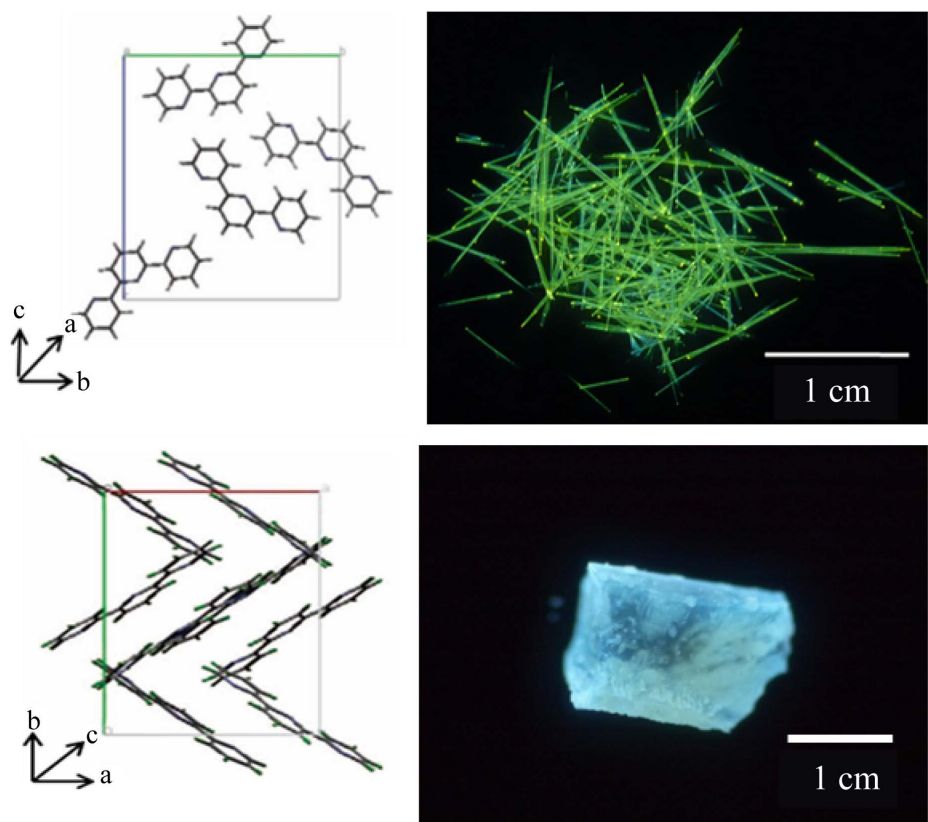

Figure 2. Crystal structures of orthorhombic (upper) and monoclinic (bottom) forms of $2,2^{\prime}: 6^{\prime}, 2^{\prime \prime}$-terpyridine and luminescent color when a $365 \mathrm{~nm}$ UV light was exposed to Terpy reagent.

of the Terpy especially for non-luminescent orthorhombic form was challenged.

\section{Experimental Section}

\subsection{Material}

All materials used in this study were obtained from commercial sources. 2,2':6',2"-Terpyridine (purity > 98.0\%) was obtained from Tokyo Chemical Industry. Some unidentified impurities are included in the Terpy reagent. To identify the impurity species and to know their effects on luminescent properties of Terpy crystals, purification of the Terpy reagent was carried by column chromatography using following materials.

Stationary phase: silica gel (Wakosil C-300, Wako Pure Chemical Industries) or activated alumina (Wako Pure Chemical Industries).

Mobile phase: hexane (purity > 95.0\%, Wako Pure Chemical Industries), tetrahydrofuran (THF, purity > 99.5\%, Wako Pure Chemical Industries).

\subsection{Method}

In this study, purification of Terpy reagent is performed and then evaluation of photoluminescence properties of the resultant crystals, total four type, were performed.

Isolation of impure species included originally in the Terpy reagent was performed by column chromatography. An example of the method is shown in Figure 3. In the Terpy reagent, at least two impure species existed as shown later, thus two different types of adsorbents, activated alumina and silica-gel, were used as the statio- 


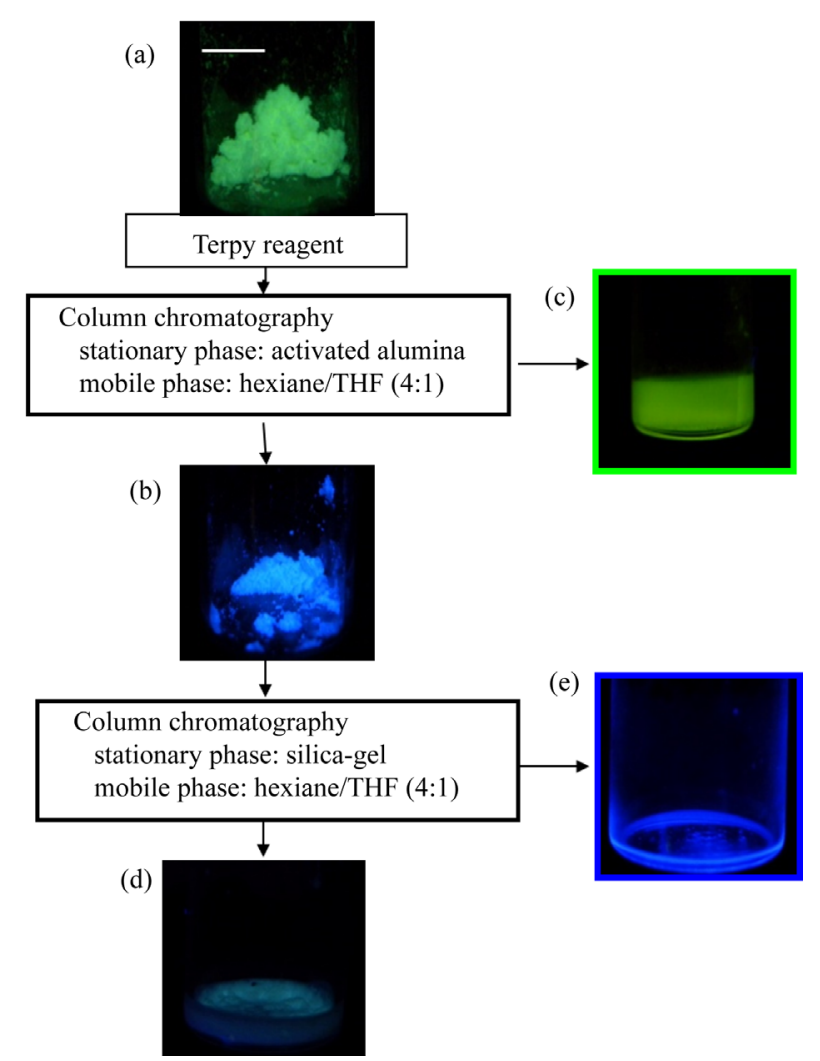

Figure 3. Flow diagram of purification of reagent Terpy by chromatography and emission of substances that were separated by chromatography.

nary phase. In case of Figure 3, activated alumina was packed in the vertical glass tube. Terpy reagent was dissolved into THF/hexane (1:4 in molar ratio) solvent and this mobile phase was added from the top of the column and impure phase was isolated. In the next step, the stationary phase was replaced by silica-gel and the Terpy remained solution was added to the column for isolation of another impurities.

By this two-step column chromatography, we have obtained at least two type of impurities in addition to pure Terpy. Structure of the impurities were analyzed using following method:

Fluorescence spectra (FP-6500, JASCO), gas chromatography-mass spectrometry. (GCMS-QP2010, Shimazu), nuclear magnetic resonance (NMR, AV400M, Bruker) and FT-IR spectrometry (FT/IR4200typeA, JASCO). Crystal structure of the Terpy crystals were identified by Powder X-ray diffraction (RINT 2200, CuK $\alpha, 1.5418$ A).

\section{Results and Discussion}

\subsection{Purification of Terpy and Identification of Impurities}

Figure 3(a) shows Terpy reagent (orthorhombic form) irradiated with UV lamp having 365 nm wavelength. By the literature data [3], orthorhombic form of Terpy have maximum emission wavelength (353 nm) in invisible region. However, the reagent crystals used in this study emitted strong green color light. This result suggests that impure species included in the reagent is luminescent and combination of the impurity species in the host framework of Terpy cause the such emission as shown in Figure 3(a). Using the activated alumina, green lightemitting impurity, named as 'impurity G' hereafter, was isolated as shown in Figure 3(c). In the remained solution, Terpy is existed and recovery of Terpy was carried by recrystallization. Figure 3(b) is a product of the recrystallization. The recrystallized crystals showed orthorhombic form of Terpy, however, the crystals emit blue light that is different emission color compared with the literature data. Some blue-light emitting impurity must remained in the crystals. Using another absorbent (silica-gel), the blue emitting impurities were successfully isolated as shown in Figure 3(e) and the remained crystals, Figure 3(d), showed very weak emission. Figure 4 


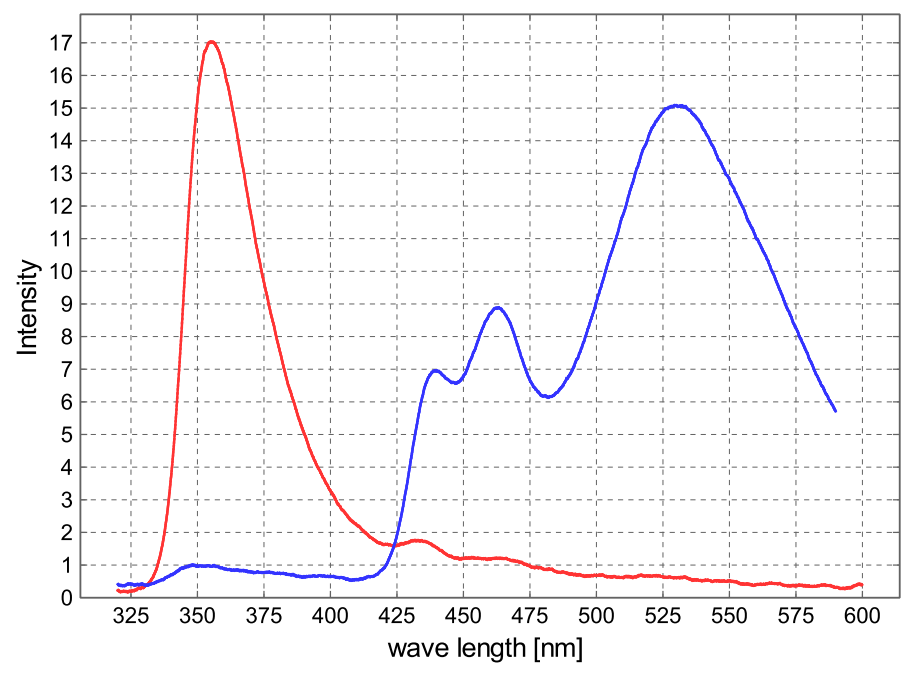

Figure 4. Emission spectrum of Tery before and after purification by two-step column chromatography. Blue line: Terpy reagent, red line: Purified Terpy.

shows emission spectrum of Terpy reagent (blue line, correspond to Figure 3(a)) and Terpy crystals after twotime purification (red line, Figure 3(d)). The former crystals showed wide range emission as shown by the blue line, while the purified Terpy showed only a single peak at around $353 \mathrm{~nm}$ agreed with that of literature data of pure Terpy. From this result, the crystals in Figure 3(d) is pure Terpy and almost all impurities were removed by the chromatography. There exists two type of impurities of blue and green color emissive impurities.

Indentification of the impurity $\mathrm{G}$ was performed with the combination of spectrum measurements.

1) GC-MS: mass spectroscopy measurement of the Green impurity showed maximum mass of $249 \mathrm{~m} / \mathrm{z}$ which is larger by $16 \mathrm{~m} / \mathrm{z}$ of mass than that of pure Terpy $(233 \mathrm{~m} / \mathrm{z})$, suggesting addition of one -OH group instead of one $-\mathrm{H}$ of pyridine ring.

2) NMR data are as follows suggesting existence of Terpy skeleton and existence of -OH group at $14.42 \mathrm{ppm}$.

${ }^{1} \mathrm{H}$ NMR (500 MHz, CDCl $): \delta 14.42(\mathrm{~s}, 1 \mathrm{H}), 8.79(\mathrm{~d}, 1 \mathrm{H}, J=8 \mathrm{~Hz}), 8.65(\mathrm{~d}, 1 \mathrm{H}, J=5 \mathrm{~Hz}), 8.54(\mathrm{~d}, 1 \mathrm{H}, J=5$ $\mathrm{Hz}$ ), 8.49 (d, $1 \mathrm{H}, J=8 \mathrm{~Hz}$ ), 8.40 (d, $1 \mathrm{H}, J=9 \mathrm{~Hz}$ ), 7.96 (ddd, $1 \mathrm{H}, J=7.8,7.8,2 \mathrm{~Hz}$ ), 7.82 (ddd, $1 \mathrm{H}, J=7.8$, $7.8,2 \mathrm{~Hz}$ ), 7.45 (d, $1 \mathrm{H}, J=9 \mathrm{~Hz}$ ), 7.37 (ddd, $1 \mathrm{H}, J=6.3,6.3,1 \mathrm{~Hz}$ ), 7.26 (ddd, $1 \mathrm{H} J=6,6,1 \mathrm{~Hz}$ )

${ }^{13} \mathrm{C}$ NMR (500 MHz, CDCl3): $\delta 158.32,157.20,156.22,148.95,146.49,145.40,137.85,136.65,135.44$, 126.46, 123.66, 123.02, 122.78, 121.94, 120.27

3) FT-IR measurements showed also existence of Terpy in addition existence of -OH vibrational mode at around $3400 \mathrm{~cm}^{-1}$.

From the above results, addition of -OH group to Terpy as shown in Figure 5, [2,2':6',2"-Terpyridine]-3'-ol (TerpyOH hereafter)] is the identity of the green impurity. From the chemical structure of TerpyOH, such a strong emission may be explained as follows. For pure Terpy molecule, since the pyridine rings are linked by a single bond, the ring may be rotate easily. Even if the molecules are excited, the energy are released as thermal motion. However, for TerpyOH, intramolecular hydrogen bond may form as shown by dotted line in Figure 5. Thus rotational movement of the pyridine rings is limited by the hydrogen bond. This cause excited energy transfer as emission energy. Concentration of the impurity $\mathrm{G}$ in the reagent Terpy crystals have been estimated as Terpy:impurity $\mathrm{G}=571: 1$ in molar ratio. Such a trace amount of impurity $\mathrm{G}$ enhance the green emitting. This is surprising result. In other word, only a trace amount of emitting molecule is required for light emitting device.

We have applied the same methodology for the blue impurities, the results were complicated and was impossible to be identified. The resultant blue impurities must be non-single components.

\subsection{Photoluminescent Properties of Four Types of Terpy Crystals}

Now we have isolated three Terpy related compounds of pure Terpy, Green emitter TerpyOH and blue impurities (still unknown structure). Usually, luminescent properties of a compound have been studied using a pure compound and great efforts have been paid for the purification. We know that, however, we should point out 
<smiles></smiles>

Figure 5. Chemical structure of 2,2':6',2"-Terpyridine-3'-ol as a source of green light emitter.

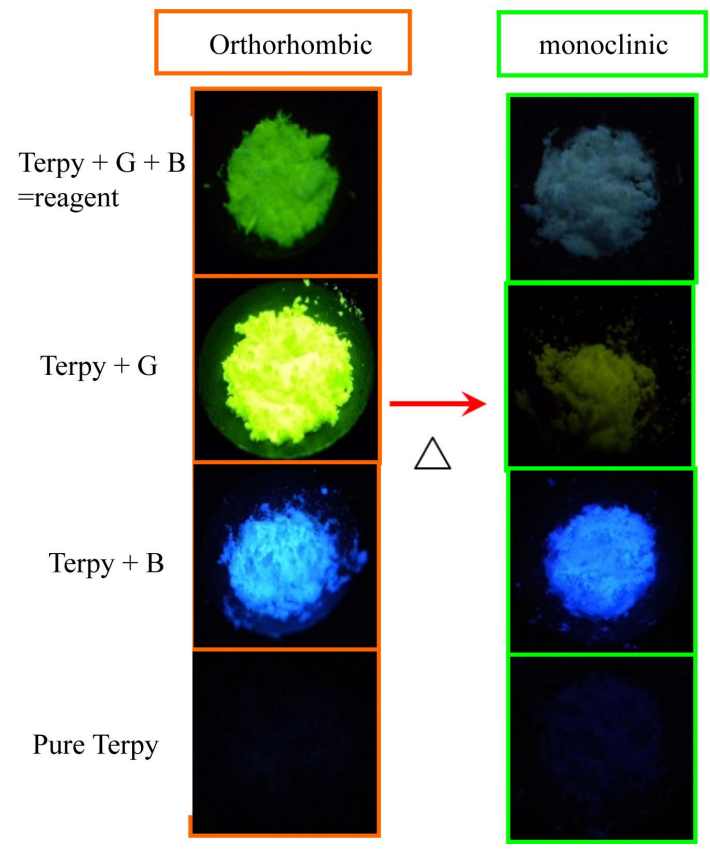

Figure 6. Luminescent color of the various type of Terpy crystals. (left side: orthorhombic form): Terpy reagent (top) = Terpy + green impurity (TerpyOH) + blue impurity. Second and third photo from the top are the pure Terpy + impurityGreen, pure terpy + impurity Blue. The bottom is pure terpy. On heating the orthorhombic crystlas, lluminescence properties were changed as right side.

undetectable amount of traces of impurity enhanced the emission in case of Terpy. This is very interesting. Thus we have actively utilize impurities as color emitter in contrast to the usual method. Combination of these substances may lead to multi-color emission.

Figure 6 shows luminescent color of the various type of Terpy crystals. At first luminescent color of the crystals (left side pictures) are examined. Terpy reagent (top) that is mixture of Terpy and traces of green impurity (TerpyOH) and blue impurity. Second and third photo from the top are the pure Terpy + traces of impurityG, pure terpy + traces of impurity Blue. The bottom is pure Terpy which shows weak luminescence in visible region. From these results, luminescent color tuning of Terpy is possible by changing the doping luminescence enhancer. In addition, the emission of orthorhombic form may be changed by transformation by heating (about $85^{\circ} \mathrm{C}$ ). Except for blue emitter, intensity of light became low.

\section{Conclusion}

The Terpy reagent in orthorhorhombic form crystal emits strong light by the doping effect of some impurities. One of the impurities was identified as green emitter, 2,2':6',2"-Terpyridine-3'-ol. Existence of the other blue emitter impurities was also recognized. Multi-color luminescence of the crystals was succeeded by combination of pure Terpy with the impurities. 


\section{References}

[1] Bessel, C.A., See, R.F., Jameson, D.L., Churchill, M. and Takeuchi, K.J. (1992) Structural Considerations of Terdentate Ligands: Crystal Structures of 2,2': 6',2'-Terpyridine and 2,6-bis(pyrazol-1-yl)pyridine. Journal of the Chemical Society, Dalton Transactions, 22, 3223-3228. http://dx.doi.org/10.1039/DT9920003223

[2] Bowes, K.F., Clark, I.P., Cole, J.M., Gourlay, M., Griffin, A.M.E., Mahon, M.F., Ooi, L., Parker, A.W., Raithby, P.R., Sparkes, H.A. and Towrie, M. (2005) A New Polymorph of Terpyridine: Variable Temperature X-Ray Diffraction Studies and Solid State Photophysical Properties. CrystEngComm, 7, 269-275. http://dx.doi.org/10.1039/b502275d

[3] Mutai, T., Satou, H. and Araki, K. (2005) Reproducible On-Off Switching of Solid-State Luminescence by Controlling Molecular Packing through Heat-Mode Interconversion. Nature Materials, 4, 685-687.

http://dx.doi.org/10.1038/nmat1454 